\title{
IDENTIFICAÇÃO DAS DIFICULDADES À IMPLEMENTAÇÃO DA POLÍTICA DE REDUÇÃO DE DANOS NO TRATAMENTO DE USUÁRIOS DE ÁLCOOL E OUTRAS DROGAS NO BRASIL: UMA REVISÃO INTEGRATIVA
}

\author{
IDENTIFICATION OF THE DIFFICULTIES FOR THE \\ IMPLEMENTATION OF HARM REDUCTION TREATMENT POLICY \\ FOR USERS OF ALCOHOL AND OTHER DRUGS IN BRAZIL: AN \\ INTEGRATIVE REVIEW
}

\author{
Pedro De Simone Caixeta \\ Matheus Vinicius de Araújo Lucena \\ Maria Eduarda Sá Abu Adiya
}

\begin{abstract}
RESUMO: OBJETIVO: Identificar, na literatura existente, quais são as principais dificuldades que retardam a implementação efetiva da política de redução de danos (RD) com foco no tratamento de usuários de álcool e outras drogas no Brasil. METODOLOGIA: Uma revisão integrativa. A pesquisa foi realizada nas bibliotecas virtuais BVS e SciELO. Os descritores utilizados foram "Redução do Dano" AND "Brasil". Selecionaram-se artigos publicados entre 2009 e 2019 que estivessem disponíveis na forma online com texto completo e cujo assunto principal fosse a estratégia de redução de danos. Foram excluídos teses, revistas literárias, monografias e artigos que divergiam da temática. Encontraram-se dez artigos na BVS e cinco artigos na SciELO, sendo um deles comum a ambas as bibliotecas, totalizando quatorze artigos selecionados. RESULTADOS: As principais dificuldades identificadas nos artigos selecionados foram: preconceito contra usuários de álcool e outras drogas e contra a política de RD; falta de preparo dos profissionais diante dos usuários de álcool e outras drogas; problemas de ordem política; produção científica inficiente sobre a eficácia da política de RD; predomínio da ideologia proibicionista; desvalorização dos redutores de danos e problemas de ordem social. CONCLUSÃO: Devido aos diversos obstáculos à implementação da política de RD no Brasil e reconhecendo sua maior eficácia, deve-se repensar as políticas públicas existentes para a sua consolidação. Observa-se, assim, a necessidade de aprimorar a formação dos profissionais e tornar as políticas mais universais e duradouras, a fim de fortalecer a rede de proteção e os cuidados aos usuários.
\end{abstract}

Palavras chave: Brasil. Drogas llícitas. Política Pública. Redução do Dano. Saúde Pública. 
ABSTRACT: OBJECTIVE: to identify what are the main difficulties that delay the effective implementation of harm reduction (HR) policy in the existing literature focusing on the treatment of users of alcohol and other drugs in Brazil. METHODOLOGY: an integrative review. The research was conducted in the BVS and SciELO virtual libraries. The descriptors used were "Harm Reduction" AND "Brazil". Articles published between 2009 and 2019 that were available online, in full text and whose main subject was the harm reduction strategy were selected. Theses, literary magazines, monographs and articles that differed from the theme were excluded. The research identified ten articles in the BVS and five articles in SciELO virtual libraries, one of which being common to both platforms, totaling fourteen selected articles. RESULTS: the main difficulties identified in the selected articles were: prejudice against users of alcohol and other drugs and against HR policy; lack of preparation of professionals in dealing users of alcohol and other drugs; public policy problems; insufficient scientific production on the effectiveness of HR policy; predominance of prohibitionist ideology; devaluation of harm reducers and problems of social nature. CONCLUSION: due to the various obstacles regarding the implementation of HR policies in Brazil and recognizing its greater effectiveness, existing public policies for its consolidation should be rethought. Thus, there is a need to improve the training of professionals and turning public policies more universal and long lasting in order to strengthen the safety network and user care.

Keywords: Brazil. Street Drugs. Public Policy. Harm Reduction. Public Health. 


\section{INTRODUÇÃO}

O uso de substâncias psicoativas pela humanidade é tão antigo quanto a sua própria existência, seja por finalidades recreativas, religiosas ou terapêuticas. Esse uso veio acompanhado de determinadas regulações sociais, que, através de normas e convenções, estabeleciam como, quando e em qual quantidade tais drogas poderiam ser utilizadas. Com $o$ advento da revolução industrial e com a transformação da droga em mercadoria, houve uma expansão de seu consumo e uma perda significativa de parte da regulação sócio-cultural existente. A perda desse lastro cultural que regulava o consumo de drogas trouxe problemáticas sociais e de saúde, tornando-se necessário recorrer ao Estado como novo intermediador, através da formulação e da implementação de políticas públicas (ALVES, 2009).

Nesse contexto, há dois grandes posicionamentos políticos sobre como 0 Estado deve enfrentar as questões relacionadas ao consumo de drogas: a abordagem do proibicionismo e a de redução de danos (RD). A primeira tem um forte caráter moral e repressivo, baseado na criminalização do consumo e na noção de "guerra às drogas", focando suas ações na diminuição da demanda e da oferta de drogas ilícitas. Já a segunda tem por objetivo a minimização dos danos associados ao uso, sem necessariamente coibi-lo (ALVES, 2009). Tais abordagens baseiam-se em modelos teóricos de gestão e de compreensão do fenômeno das drogas. Segundo Trad et al. (2015), enquanto a perspectiva proibicionista norteia-se pelos modelos penal e médico, a abordagem da RD leva em conta o modelo sociocultural (apud ROMANí, 2004).

O modelo penal, ou jurídico-moral, entende que a utilização de drogas constitui um ato moralmente errado e que, portanto, deve ser configurado como delito, criminalizando o usuário. A questão das drogas é, dessa forma, compreendida como problema de segurança pública e o encarceramento em massa passa a ser sua solução. No Brasil esse modelo foi instituído através do decreto 4.294 do Código 
Penal Brasileiro de 1924, que definia a pena de prisão para quem comercializasse drogas ilícitas.

Já o modelo médico, ou de doença, segundo Trad et al. (2015), encara a dependência como um estado patológico, em uma transposição do esquema de doença contagiosa (APUD NOWLIS, 1977). Tal modelo só passou a ser considerado no Brasil após a promulgação da lei no 5726 de 1971, tornando-se a concepção médico-psiquiátrica preponderante (MACHADO, 2006). Segundo Lermen et al., (2014), o modelo médico garantiu subsídios científicos para legitimar o controle sobre o uso de drogas, fazendo das internações hospitalares uma alternativa ao controle dos "infratores viciados" (APUD ALVES, 2009).

Apesar das diferenças entre os modelos, ambos possuem um forte caráter repressivo e admitem a eliminação total do consumo e a abstinência como exclusiva meta do tratamento (ALVES, 2009).

\section{Política de Redução de Danos}

Para a política de RD, o estado de abstinência é uma das possibilidades clínicas, a depender da vontade do usuário. Ela não é contrária à abstinência como meta possível, mas à abstinência como regra absoluta (SOUZA, 2013). Entende-se que a recusa do paciente a se tornar abstinente nunca deveria ser motivo para a exclusão do tratamento (DIAS et al., 2003). A RD trabalha com a noção de pragmatismo empático, ao contrário do idealismo moralista do proibicionismo, por entender que o comportamento prejudicial acontece e que se deve assumir a resolução mais pragmática, sem distinção moral. Por não exigir a abstinência total, a $\mathrm{RD}$ é conhecida como uma política de baixa exigência, fator de fixação do dependente à terapêutica, em contraposição às estratégias proibicionistas de alta exigência, que servem como um fator de exclusão e marginalização do usuário. De acordo com Machado e Boarini (2013), a RD tende, portanto, a uma formatação mais humanitária, uma vez que entende que os serviços de saúde devem chegar ao usuário, e não o contrário, compreendendo as vulnerabilidades do indivíduo (APUD 
DIAS et al., 2003). Além disso, entende-se que a política de RD tem base comunitária e é realizada de baixo para cima, permitindo ao usuário participar do seu tratamento, construído em conjunto com ele (DIAS et al., 2003). Por ser mais flexível, a PRD é, consequentemente, mais eficaz. Segundo Machado e Boarini (2013), dados do ministério da saúde indicam que "23\% dos usuários atendidos pelos programas de redução de danos procuram tratamento para a dependência química" (APUD CARLINI, 2003, p. 366).

Visto que prioriza as especificidades do contexto local e a história do paciente, declarando-se contrária à homogeneização da vida, não se pode dizer que haja um conjunto de ações preestabelecidas que possam ser classificadas como estratégias de RD, apenas como indicadores (MACHADO; BOARINI, 2013). Além dos grupos que rechaçam por completo o modelo de $\mathrm{RD}$, há aqueles que têm uma aceitação parcial da abordagem, pautados no comportamento de risco: acreditam que ela deva se resumir à diminuição da transmissão de doenças e agravos, mas que a finalidade clínica continue sendo a abstinência (OLIVEIRA et al., 2019). Tais posicionamentos ainda são marcados por um forte caráter moral, e não pela falta de cientificidade da estratégia como muitos alegam, pois diversas experiências nacionais e internacionais já validaram a comprovação científica da abordagem de RD (MACHADO; BOARINI, 2013).

Dessa forma, fica claro que, apesar da política de Redução de Danos estar ganhando cada vez mais espaço nas discussões de saúde pública, existem dificuldades que impedem sua implementação efetiva e que devem ser superadas. $O$ presente trabalho tem como objetivo identificar quais são essas dificuldades, levando em consideração o contexto da política de RD no Brasil.

\section{METODOLOGIA}

O presente artigo trata-se de uma revisão integrativa da literatura vigente acerca do tema da RD no contexto brasileiro e das dificuldades encontradas para sua implementação. A revisão integrativa foi compreendida como o melhor método 
de pesquisa para o estudo, uma vez que abrange uma ampla quantidade de artigos e permite uma boa síntese. Além disso, através da reunião de resultados de pesquisas prévias é possível gerar novos conhecimentos, dando suporte para práticas clínicas mais efetivas.

A partir da observação da temática em questão, surgiu um questionamento: apesar da política de RD ter comprovadamente uma maior eficácia, em comparação a outras abordagens, por que ela ainda é pouco aplicada? A questão norteadora do trabalho foi então estabelecida como: quais são as dificuldades enfrentadas para a aplicação efetiva da política de RD no Brasil?

Foram selecionados artigos através das seguintes bibliotecas virtuais: Biblioteca Virtual em Saúde (BVS) e Scientific Electronic Library Online (Scielo). A pesquisa foi realizada utilizando os descritores "Redução do Dano" e "Brasil" cadastrados na plataforma DeCS da BVS, conectados pelo booleano "AND". Foram selecionados artigos publicados entre 2009 e 2019. Os critérios de inclusão foram: estudos que abordassem a temática proposta, que estivessem dentro do recorte temporal definido, que possuíssem texto completo e que estivesse disponível de forma online. Os critérios de exclusão foram: publicações do tipo tese e que não se enquadrassem em quaisquer dos critérios de inclusão. Foram encontrados artigos em português, inglês e espanhol. A pesquisa foi realizada no mês de dezembro de 2019.

A partir da pesquisa, foram selecionados cinco artigos, entre os encontrados na Scielo, e dez artigos, na BVS, um deles encontrado em ambas as bibliotecas. Sendo assim, foram selecionados quatorze diferentes artigos que se enquadraram nos critérios propostos.

\section{RESULTADOS E DISCUSSÃO}

Na tabela 1, estão listados os 15 artigos selecionados para análise, dos quais foram destacados o título, o ano de publicação e a biblioteca da qual foram retirados. Apesar das diferentes metodologias e dos objetivos cujo foco não aborde os 
impasses para a consolidação da política, todos os artigos analisados evidenciaramnos, os quais foram categorizadas e relacionadas na tabela 2, juntamente com os respectivos objetivos e metodologias utilizadas em cada um deles.

Tabela 1 - Artigos selecionados para análise, contendo título, ano de publicação e biblioteca de pesquisa.

\begin{tabular}{|c|c|c|c|}
\hline $\mathbf{N}^{\circ}$ & TíTULO & ANO & BIBLIOTECA \\
\hline 01 & $\begin{array}{l}\text { Atenção à saúde de usuários de álcool e outras drogas } \\
\text { no Brasil: convergência entre a saúde pública e os } \\
\text { direitos humanos }\end{array}$ & 2012 & BVS \\
\hline 02 & $\begin{array}{l}\text { Cenas de uso de crack na capital de Santa } \\
\text { Catarina/Brasil: a (in) visibilidade do usuário }\end{array}$ & 2019 & ScIELO \\
\hline 03 & $\begin{array}{l}\text { Drogadição no cárcere: questões acerca de um } \\
\text { projeto de desintoxicação de drogas para pessoas } \\
\text { privadas de liberdade }\end{array}$ & 20 & BVS \\
\hline 04 & $\begin{array}{l}\text { Implementation of harm reduction toward crack users in } \\
\text { Brazil: barriers and achievements }\end{array}$ & 2012 & BVS \\
\hline 05 & $\begin{array}{l}\text { Los retos de la reducción de daños como política } \\
\text { pública de drogas en Brasil }\end{array}$ & 2015 & BVS \\
\hline 06 & $\begin{array}{l}\text { Mapeando programas de redução de danos da Região } \\
\text { Metropolitana de Porto Alegre, Rio Grande do Sul, } \\
\text { Brasil }\end{array}$ & 2009 & BVS \\
\hline 07 & $\begin{array}{l}\text { Modelos de atenção à saúde de usuários de álcool e } \\
\text { outras drogas: discursos políticos, saberes e práticas }\end{array}$ & 2009 & BVS / Sc \\
\hline 08 & $\begin{array}{l}\text { O alcoolismo, suas causas e tratamento nas } \\
\text { representações sociais de profissionais de Saúde da } \\
\text { Família }\end{array}$ & 2015 & BVS \\
\hline 09 & $\begin{array}{l}\text { Percepção de pessoas que usam drogas acerca do } \\
\text { tratamento em um Centro de Atenção Psicossocial }\end{array}$ & 2018 & SciELO \\
\hline 10 & $\begin{array}{l}\text { Políticas de redução de danos no Brasil: contribuições } \\
\text { de um programa norte americano }\end{array}$ & 2014 & BVS \\
\hline 11 & $\begin{array}{l}\text { Políticas sobre drogas no Brasil: a estratégia de } \\
\text { redução de danos }\end{array}$ & 2013 & BVS \\
\hline 12 & $\begin{array}{l}\text { Práticas emancipatórias na área de drogas: construção } \\
\text { de projetos com trabalhadores da Atenção Primária à } \\
\text { Saúde }\end{array}$ & 2019 & SciELO \\
\hline 13 & $\begin{array}{l}\text { Redução de danos numa instituição militar de } \\
\text { recuperação de dependentes químicos: desafios e } \\
\text { possibilidades }\end{array}$ & 2015 & BVS \\
\hline 14 & $\begin{array}{l}\text { Uso de drogas injetáveis entre mulheres na Região } \\
\text { Metropolitana de Santos, São Paulo, Brasil }\end{array}$ & 2016 & SciELO \\
\hline
\end{tabular}

Fonte: produzido pelos autores. 
Tabela 2 - Resumo dos artigos selecionados, contendo objetivo, metodologia e resultados (continua).

\section{No AUTORES OBJETIVOS METODOLOGIA RESULTADOS}

01 ALVES, V.Avaliar o cuidadoRevisão integrativaEscassez de produção S. et al. com dependentes da literatura científica

02 \begin{tabular}{ll} 
ZEFERINO & cena do uso de ${ }_{\text {crack }}$ \\
\hline
\end{tabular} Descrever $\quad$ as Avaliação qualitativa Preconceito contra 03 LERMEN, Analisar um projetoAvaliação qualitativaPreconceito contra H. S. et al. de RD do projeto de RD usuários e a política Preconceito contra 04 O, A. et al.

Avaliar programas brasileiros redução de danos redução de danos usuários e a política; Falta de preparo profissional; roblemas políticos

Tratar as principais Análise de 15Problemas políticos; M. et al. da redução de $\begin{array}{lll}\text { no Brasil } & & \begin{array}{c}\text { saúdeDesvalorização } \\ \text { redutores de danos }\end{array}\end{array}$

Discutir cincoEstudo em estadosusuários e a política; Falta que implementaramde preparo profissional; deprograma piloto deProblemas políticos; desvalorização dos redutores de danos Preconceito contra 06 NARDI, H.sustentabilidade emapeamento de 11 usuários e a política; C. et al. características dosprogramas políticos; programas de RD de redução de danos $D$ esvalorização dos redutores de danos

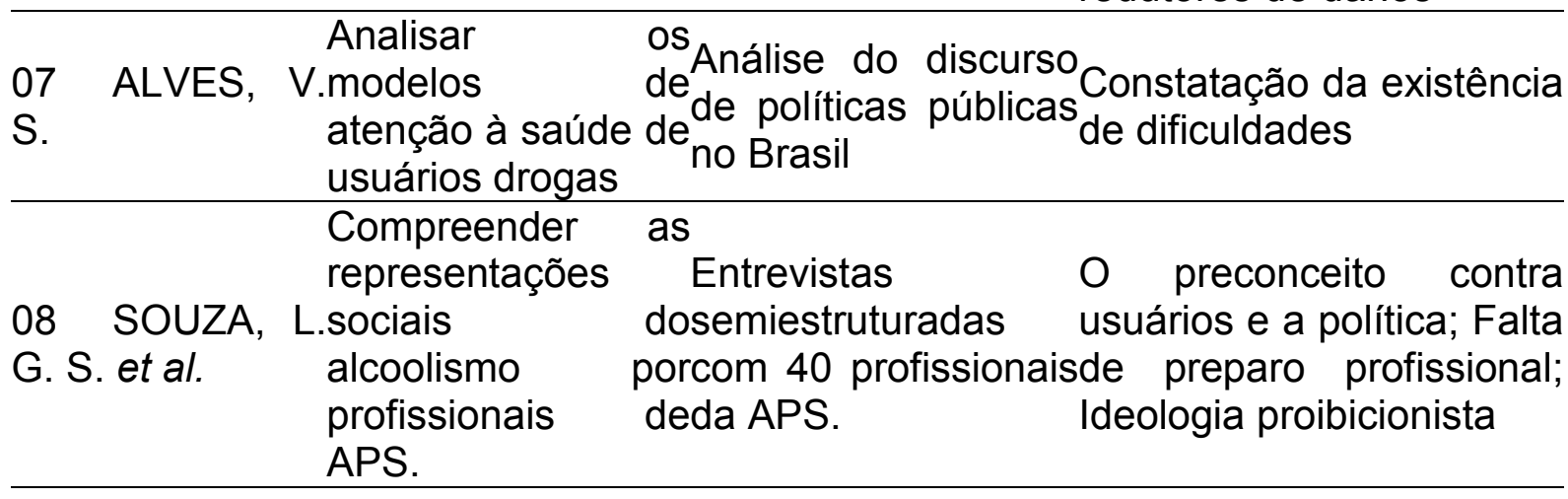

Entender

09 MANENTE, percepção V. et al.
aEntrevista realizada doscom 21 usuários de usuários sobre seuálcool e outras tratamento drogas \begin{tabular}{ll}
\hline 10 DIAS, A. I. & Discutir as práticas \\
Et al. & Revisão narrativa daDesvalorização \\
atenção aos UDI & literatura
\end{tabular} dos 
11 MACHADO,Relatar o históricoPesquisa

L. V. et al. da RD no Brasil bibliográfica

Escassez de produção Criar projetos paraPesquisa qualitativa,

12 OLIVEIRA, a implementarrealizada por meio científica

L. C. et al. práticas de uma emancipatórias naconversa roda de Desvalorização dos área de drogas trabalhadores

13 GOMES, Descrever A. M. S. etimplantação al. práticas de RD

aPesquisa qualitativaO preconceito contra deem uma unidadeusuários e a política; $A$ hospitalar militar ideologia proibicionista

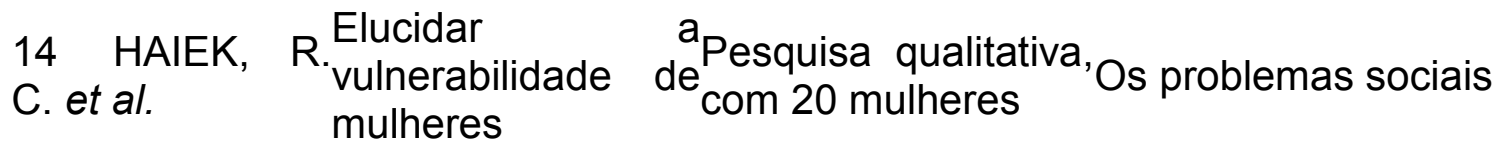

Fonte: produzido pelos autores.

É um fato que a política de redução de danos encontra algumas dificuldades para a sua implementação definitiva (ALVES et al., 2009). Dentre elas, as principais que foram analisadas pelos artigos selecionados foram: preconceito contra os usuários de álcool e outras drogas e contra a política de RD; falta de preparo dos profissionais diante dos usuários de álcool e outras drogas; problemas de ordem política; produção científica insuficiente sobre a eficácia da política de RD; predomínio da ideologia proibicionista; desvalorização dos redutores de danos; problemas de ordem social; entre outras.

\section{Preconceito contra usuários de álcool e outras drogas e contra a política de RD}

Problemática trazida por seis dos artigos analisados, o preconceito contra usuários de álcool e outras drogas está presente de forma significativa entre as equipes de saúde. Souza et al. (2015) destacaram que as equipes de Saúde de Família têm preconceito com usuários de álcool, que é uma droga lícita e aceita socialmente. Essa situação é ainda mais dura quando se trata de usuários de drogas 
ilícitas, em função da visibilidade negativa que eles têm na sociedade (NARDI et al., 2009).

Diante disso, colocar em prática as ações de Redução de Danos se torna um desafio, uma vez que se tratam de condutas que são consideradas fora do padrão de legalidade pelas autoridades (LERMEN et al., 2014). Por se tratarem de práticas que vão de encontro às estratégias focadas na abstinência já consolidadas e aceitas socialmente, cria-se uma dificuldade extra de negociação para apoio político e financeiro, o que compromete a sustentabilidade dos programas de RD (NARDI et al,. 2009).

Devido a esse estigma, os usuários de álcool e outras drogas resistem em se reconhecerem dependentes, o que dificulta sua adesão aos programas de RD, uma vez que, para eles, isso simboliza ser doente e fraco (GOMES et al., 2015).

Domanico et al. (2012) destacaram que, mesmo onde a RD já é praticada, o estigma do usuário como doente e criminoso persiste, pois, fora das áreas de RD, continuam sendo vistos como pessoas envolvidas em práticas ilícitas e que necessitam de tratamento obrigatório. Além disso, são comuns casos de assédio policial, que confiscam ou quebram equipamentos empregados para a RD.

No mais, é muito comum vivenciar forte oposição da mídia (DOMANICO et al., 2012), marcada por um posicionamento contrário às políticas de redução de danos, uma vez que tende a reforçar certos estereótipos, tratamentos e políticas, legitimando e perpetuando ações de caráter excludente (ZEFERINO et al., 2019).

\section{Falta de preparo dos profissionais}

Esse ponto foi apresentado em três dos artigos selecionados, sobre o qual os próprios funcionários das USF reconhecem que a dificuldade está em sua formação, uma vez que estão mais preparados para lidar com doenças orgânicas, do que com usuários em situação de drogadição (SOUZA et al. 2015). 
Já em relação aos voluntários dos programas de RD, Domanico et al. (2012) destacaram que há uma importante falta de treinamento formal e de supervisão desses trabalhadores, sobretudo os que atuam em campo.

No estudo de Zeferino et al. (2019), chamou atenção o fato de os profissionais não abordarem os usuários de crack quanto aos cuidados necessários, evidenciando que estes atores não têm atuado frente a esta realidade de acordo com a exigência das políticas públicas existentes.

\section{Problemas de ordem política}

Esse aspecto foi observado em quatro dos artigos selecionados. Diz respeito a uma situação decorrente, sobretudo, do desinteresse da classe política em apoiar uma prática que não é bem aceita socialmente. Cria-se, assim, uma situação paradoxal, pois os programas de RD precisam do apoio político, ao mesmo tempo em que lutam contra uma política antidrogas hegemônica na maquinaria governamental (MORAES et al., 2015).

Nesse aspecto, é importante destacar que, apesar do avanço considerável no incentivo político, esse apoio ainda é insuficiente para garantir a continuidade a implementação de ações de RD na rede de saúde (NARDI et al., 2009). Além disso, Nardi et al., (2009) também destacaram que a maior parte dos incentivos está atrelada às políticas de DST/AIDS, enquanto que uma minoria é voltada à saúde mental dos usuários de álcool e outras drogas.

Outro importante problema relatado pela maioria dos gerentes de programas de RD foi a descontinuidade dos fundos (DOMANICO et al., 2012). Isso é decorrente da troca de gestão, sobretudo municipais, o que gera uma instabilidade no apoio político, fato que é observado apenas nos programas e ações de RD governamentais (NARDI et al., 2009).

Além dos problemas que dificultam a consolidação dos centros de RD, é possível observar diversos outros estruturais acerca de políticas públicas para 
usuários de drogas para encarar, com seriedade, esse impasse como problema de saúde pública (ZEFERINO et al., 2019).

Zeferino et al. (2019) também destacaram que é notório que o usuário de crack se mostrou invisível aos olhos da polícia, instituições de saúde, assistência social e da comunidade civil.

\section{Predomínio da ideologia proibicionista}

Esse tema foi abordado por três dos artigos selecionados. Trata-se de uma situação marcante quando se fala, sobretudo, a respeito do álcool, uma droga lícita, pois se adotou a ideia de que um alcoólico sempre será um alcoólico e de que a única solução é a abstinência total e definitiva, discussão que reproduz entre os profissionais de saúde, inclusive, que enquadram o caso do alcoolismo como "tudo ou nada" (SOUZA et al., 2015).

No estudo de Manente et al (2018), foi exposto que a forma de tratamento que os participantes mais buscam no CAPS é a abstinência, estratégia que não tem se mostrado como a mais eficiente, ainda assim, é tida como a melhor alternativa. Consoante a isso, alguns participantes não acreditam na efetividade da redução de danos, usando como uma das formas de justificativa o descontrole no consumo.

Nesse sentido, outro fator complicador, relatado pelos usuários, é que existe uma resistência quando não estão preparados para continuar com a abstinência, decisão que muitas vezes não é bem aceita (GOMES et al., 2015).

\section{Desvalorização dos redutores de danos}

Cinco dos artigos selecionados, destacaram que, sobre os redutores de danos, um dos principais problemas é a marcante precarização dos vínculos de trabalho, além do fato de esses trabalhadores não contarem com formação 
especializada (DIAS et al., 2014). Na maioria dos casos, eles são contratados como autônomos, ou seja, não gozam das garantias da legislação trabalhista, exceto daqueles que são cedidos pela prefeitura (NARDI et al., 2009).

Os redutores de danos são uma categoria profissional marginalizada, composta por profissionais híbridos, considerados inadequados para as exigências da saúde pública (MORAES et al., 2015). Diante disso, existe uma grande dificuldade em se garantir a segurança desses trabalhadores (DOMANICO et al., 2012), os quais estão sempre sujeitos a repressão policial e conflitos locais (DIAS et al., 2014).

Diante disso, quando perguntados, os próprios redutores de danos pontuaram dificuldades vividas nos serviços tais quais: motivação de profissionais para além da realização das metas quantitativas, exigidas pelos planos diretores municipais; alta rotatividade dos trabalhadores nos serviços primários; e preferência pelo atendimento hospitalar/médico (OLIVEIRA et al., 2019).

\section{Problemas de ordem social}

Esse aspecto foi identificado em um artigo entre os selecionados. Nesse artigo, de Haiek et al. (2016), as mulheres entrevistadas possuíam a percepção de que sua forma de vida não era aceita socialmente (APUD. BRASILIANO, 2005). Elas consideraram a parceria sexual e o relacionamento com os UDI prejudiciais, visto que estimulavam um maior consumo de drogas, pelo acesso contínuo e exposição frequente (HAIEK et al., 2016). Essa situação acaba repercutindo na adesão aos programas de redução de danos, na medida em que, pela submissão ao homem, dificilmente uma mulher participará do tratamento se seu companheiro a recusa. Isso deve-se a uma rede estrutural de violência contra a mulher (APUD DANTASBERGER; GIFFIN, 2005; APUD REICHENHEIM; DIAS; MORAIS, 2006). 


\section{Produção científica insuficiente sobre a eficácia da redução de danos}

Esse tema é mencionado em dois dos artigos analisados. Diz respeito a importância de serem realizadas publicações acerca do tema da RD, para que a política ganhe mais aceitabilidade na sociedade.

No âmbito acadêmico, já é visível o rompimento com o paradigma proibicionista, seja pela aceitação total ou parcial da RD em sua substituição. No entanto, a temática não é abordada com muita clareza (MACHADO et al., 2013), o que dificulta a sua aceitação social, tendo em vista que é fundamental que as políticas de RD tenham sua eficácia comprovada por meio de referências em conhecimentos científicos. (ALVES et al., 2012).

\section{CONSIDERAÇÕES FINAIS}

As dificuldades para a implementação da Redução de Danos como principal estratégia para lidar com usuários de drogas indicam a complexidade do tema e não devem ser debatidas de forma isolada, uma vez que se influenciam conjuntamente.

O estigma sobre usuários de drogas, que os caracteriza como indivíduos transgressores, perturbadores da ordem social e passíveis de punição é o contexto por trás das dificuldades de ordem política e científica da redução de danos. Isso significa dizer que o preconceito existente em torno do tema desestimula, por exemplo, políticas governamentais voltadas para a estratégia. Assim, há menos recursos alocados para os programas de $\mathrm{RD}$, uma vez que o político que se mostrar favorável a ela corre o risco de ser mal visto por seus eleitores. Observa-se também que, no campo da produção científica, os estudos que tratam sobre RD comprovam a sua eficácia, mas ainda são insuficientes para consolidá-la como uma política hegemônica. Esse cenário está ligado à visão equivocada e estigmatizada sobre o 
uso de drogas, o que termina por perpetuar a falta de conhecimento geral sobre essa abordagem e a não-validação dos procedimentos de redução de danos.

A priorização das políticas voltadas para a abstinência ainda é a realidade e se mostra consonante com visões moralistas e patologizantes, que veem na proibição do uso de drogas a melhor solução possível. Essa abordagem desconsidera que uma sociedade totalmente sem drogas é utópica e não pressupõe a liberdade individual de fazer uso ou não de drogas. Além disso, a ideia de que a abstinência é o único caminho possível pode ser extremamente danosa para a forma como usuários de drogas se enxergam, pois, frequentemente, a frustração de não conseguir parar totalmente o uso pode ser um estímulo para que se continue usando.

Outras questões a serem enfrentadas dizem respeito à falta de preparo dos profissionais para atuarem na perspectiva da RD - principalmente profissionais de saúde, frequentemente condicionados a encarar os processos saúde-doença de forma puramente orgânica- e a desvalorização dos redutores de danos - com vínculos de trabalho muitas vezes precários.

Futuros estudos nessa área podem ter seu foco direcionado para: monitoramento epidemiológico de usuários de drogas afetados por programas de redução de danos; identificação e compreensão das principais necessidades de cada grupo que faça uso de determinada droga; comparação dos efeitos entre aplicar estratégia de abstinência e de Redução de Danos e, por fim, a análise qualitativa sobre a imagem da RD na sociedade.

Em conclusão, ressalta-se a importância da desconstrução dos paradigmas que envolvem o debate do uso de drogas e da Redução de Danos, de modo a fazêlo mais pragmático e comprometido com a realidade, sempre se pautando nos Direitos Humanos. 


\section{REFERÊNCIAS BIBLIOGRÁFICAS}

ALVES, Vânia; LIMA, Isabel. Atenção à saúde de usuários de álcool e outras drogas no Brasil: Convergência entre saúde pública e direitos humanos. Rdisan, São Paulo, v. 13, n. 3, p. 9-32, Nov. 2012/Fev.2013.

ALVES, Vânia. Modelos de atenção à saúde de usuários de álcool e outras drogas: discursos políticos, saberes e práticas. Cad. Saúde Pública, Rio de Janeiro, v. 25, n.11, p.2309-2319, nov, 2009.

DIAS, João Carlos et al. Redução de danos: posições da Associação Brasileira de Psiquiatria e da Associação Brasileira para Estudos do Álcool e Outras Drogas. J. bras. psiquiatr. v. 52, n.5, p. 341-348, 2003.

DOMANICO, Andrea; MALTA, Monica. Implementation of Harm Reduction Toward Crack Users in Brazil: Barriers and Achievements. Substance Use \& Misuse, n. 47, 535- 546, 2012.

GOMES, A.M.; ABRAHÃO, A.L.; SILVA, A.P. Redução de danos numa instituição militar de recuperação de dependentes químicos: desafios e possibilidades. J. res.: fundam. care. online. v. 7, n. 4, p. 3479-3486 out/dez 2015.

HAIEK, R.C.; MARTIN, Denise; ROCHA, F.C.M.; RAMIRO, F.S.; SILVEIRA, D.X. Uso de drogas injetáveis entre mulheres na Região Metropolitana de Santos, São Paulo, Brasil. Physis Revista de Saúde Coletiva, Rio de Janeiro, v. 26, n. 3, p. 917-937, 2016.

INGLEZ-DIAS, A.; RIBEIRO, J.; BASTOS, F.; PAGE, K.; Políticas de redução de danos no Brasil: contribuições de um programa norte-americano. Ciência \& Saúde Coletiva, v. 19, n. 1, p. 147-157, 2014.

LERMEN, Helena; DARTORA, Tamires; CAPRA-RAMOS, Carine. Drogadição no cárcere: questões acerca de um projeto de desintoxicação de drogas para pessoas privadas de liberdade. Estud. pesqui. psicol., Rio de Janeiro, v. 14, n. 2, p. 539-559, 2014.

MACHADO, Ana Regina. Uso prejudicial e dependência de álcool e outras drogas na agenda da saúde pública: um estudo sobre o processo de constituição da política pública de saúde do Brasil para usuários de álcool e outras drogas. Tese (Mestrado em saúde pública) - Faculdade de Medicina da Universidade Federal de Minas Gerais. Belo Horizonte, 2006.

MACHADO, Letícia; BOARINI, M. Lúcia. Políticas Sobre Drogas no Brasil: a Estratégia de Redução de Danos. Psicologia: Ciência e Profissão, v. 33, n. 3, p. 580-595, 2013.

MANENTE, V. B., SIQUEIRA, D. F., SOCCOL, K. L. S., ANDRES, S. C., CANABARRO, J. L., \& MORESCHI, C. Percepção de pessoas que usam drogas acerca do tratamento em um Centro de Atenção Psicossocial. Revista Portuguesa de Enfermagem de Saúde Mental, v. 20, p.27-33, 2018. Disponível em: 10.19131/rpesm.0223. Acesso em 04/01/2019.

MORAES, M.; MONTENEGRO, M.; MEDRADO, B.; ROMANÍ, O. Los retos de la reducción de daños como política pública de drogas en Brasil. Psicologia em Pesquisa, UFJF, v.9, n.2, p. 148-158, Julho-Dezembro de 2015.

NARDI, Henrique; RIGONI, Rafaela. Mapeando programas de redução de danos da Região Metropolitana de Porto Alegre, Rio Grande do Sul, Brasil. Cad. Saúde Pública, Rio de Janeiro, v. 25, n. 2, p. 382-392, fev, 2009. 
OLIVEIRA L. C, SOARES C. B, CAMPOS C. M. S, CORDEIRO L. Emancipatory drug practices: building projects with Primary Healthcare workers. Rev Esc Enferm USP. 2019; 53:e03528. Disponível em: http://dx.doi.org/10.1590/S1980-220X2018027803528. Acesso em $04 / 01 / 2019$.

SOUZA, L.G.; MENANDRO, M.C.; MENANDRO, P.R. 0 alcoolismo, suas causas e tratamento nas representações sociais de profissionais de Saúde da Família. Physis Revista de Saúde Coletiva, Rio de Janeiro, v. 25, n.4, p. 1335-1360, 2015.

Trad, S.N.S., Trad, L.A.B. e Romani, J.O. (2013). Contribuições das Ciências Sociais ao estudo sobre drogas e o diálogo com a produção nacional contemporânea. In: Maria Salete Bessa Jorge; Leny Alves Bomfim Trad; Paulo Henrique Dias Quinderé; Leilson Lira de Lima (Org.), Olhares Plurais Sobre o Fenômeno do Crack. (pp. 27-66). Fortaleza: EdUECE.

ZEFERINO, M. T, FERMO, V. C, FIALHO, M. B, KENTHI, A, BASTOS, F. I. Cenas de uso do crack na capital de Santa Catarina/Brasil: a (in)visibilidade do usuário. Texto Contexto Enferm [Internet]. 2019 [acesso 04/01/2019]; 28: e20170460. Disponível em: http://dx.doi.org/10.1590/1980-265X-TCE-2017-0460. 